\title{
Comprehension deficits among older patients in a quick diagnostic unit
}

\author{
This article was published in the following Dove Press journal: \\ Clinical Interventions in Aging \\ 16 April 2014 \\ Number of times this article has been viewed
}

\author{
Lisa Nebelin Hvidt ${ }^{1,2}$ \\ Kristian Nebelin $\mathrm{Hvidt}^{3}$ \\ Kim Madsen' \\ Thomas A Schmidt \\ 'Department of Emergency Medicine, \\ Holbæk University Hospital, Holbæk, \\ ${ }^{2}$ Department of Geriatrics, Gentofte \\ University Hospital, Copenhagen, \\ ${ }^{3}$ Division of Cardiology, Department \\ of Medicine, Holbæk University \\ Hospital, Holbæk, Denmark
}

Background: Higher prevalence of multiple illnesses and cognitive impairment among older patients pose a risk of comprehension difficulties, potentially leading to medication errors. Therefore, the objective of this study was to investigate comprehension of discharge instructions among older patients admitted to a Quick Diagnostic Unit (QDU).

Methods: One hundred and two patients discharged from the QDU answered a questionnaire covering understanding of their hospitalization and discharge plan. Patients' ability to recall discharge instructions and awareness of comprehension deficits, ie, ability to identify the misconceived information, were evaluated by comparing the questionnaires with the discharge letters. The population was divided into an older group (age $\geq 65$ years) and a younger group.

Results: The older group ( $\mathrm{n}=40$ ) was less able to recall correct medication instructions when compared to the younger group ( $54 \%$ versus $78 \%$, respectively; $P=0.02$ ). In multiple logistic regression analysis, correct recall of medication instructions was 4.2 times higher for the younger group compared to the older group (odds ratio 4.2, 95\% confidence interval 1.5-11.9, $P=0.007$ ) when adjusted for sex and education. The older patients were less aware of their own comprehension deficits, and in respect to medication instructions awareness decreased $6.1 \%$ for each additional year of age (odds ratio $0.939,95 \%$ confidence interval $0.904-0.98, P=0.001$ ) when adjusted for sex and education.

Conclusion: Older patients were less able to recall correct medication instructions and less aware of their comprehension deficits after discharge from a QDU. The findings of the present study emphasize the importance of thorough communication and follow-up when treating older patients.

Keywords: geriatrics, communication, discharge information, medication

\section{Introduction}

Comprehension difficulty features different levels of misconception: ability to recall information and awareness of comprehension deficits, ie, ability to identify the misconceived information. Higher prevalence of multiple illnesses and transient or chronic cognitive impairment among older patients pose a risk of comprehension difficulties, which may lead to medication errors and unplanned readmissions. ${ }^{1-4}$ Knowledge is lacking on older patients' potential comprehension difficulties in a Quick Diagnostic Unit (QDU).

Inadequate health literacy level influences patient comprehension and is more prevalent among older patients, likely due to age-related cognitive decline. ${ }^{5,6}$ At the same time, increasing age and patient comorbidities have been associated with unplanned hospital readmissions. ${ }^{3,7}$ Insufficient communication and lack of patient education by hospital personnel have been identified as causes of posthospital medication errors, likewise resulting in unplanned hospital readmissions. ${ }^{1,2}$

Although studies have shown that older patients have impaired ability to recall discharge information compared to younger patients, ${ }^{8}$ results on awareness of these
Correspondence: Lisa Nebelin Hvidt Department of Emergency Medicine, Holbæk University Hospital,

Smedelundsgade 60, DK-4300 Holbæk, Denmark

Tel +45 268 I II I3

Email lisanebelinhvidt@gmail.com 
comprehension deficits are limited. However, in general emergency departments (EDs) it has been shown that patients had compromised awareness of comprehension deficits when evaluating their discharge information. ${ }^{9,10}$

A QDU is a relatively new type of ward integrated with the ED. Patients admitted to the QDU have surgical and internal medicine conditions with an expected hospitalization of less than 2 days. Patient flow and need of quick diagnostic procedures in this setting may challenge older patients' comprehension.

Therefore, the objective of the present study was to investigate patient comprehension of discharge information among older patients and their awareness of their comprehension deficits in a QDU setting.

\section{Materials and methods}

\section{The survey}

We conducted a cross-sectional questionnaire study inviting at least 100 patients discharged from the QDU at Holbæk University Hospital in a 2-month period, June and July 2012.

Exclusion criteria were age below 18 years, patients being discharged from the QDU to another facility or unit, inability to speak or hear, need of a translator, not awake-aware-oriented, Glasgow coma scale below 15, and known dementia. The population was divided into two groups, an older group (age $\geq 65$ years) and a younger group (age $<65$ years).

The medical staff were informed about the objective and study design including the questionnaire before the beginning of the study. However, patients were not informed about the study until after the discharge interview was completed. Patients did not know the specific contents of the questionnaire but were informed about the overall aim of the study. They were not able to consult their discharge paper or medication list when answering the questionnaire.

Several physicians administered the postdischarge instructions. The discharge interview was conducted in the QDU, typically in a room shared by two patients. Answered questionnaires were collected before the patients left the department. Written consent was obtained from all the participants before enrollment. The study protocol underwent institutional review.

\section{The questionnaire}

The questionnaire covered basic characteristics, self-assessed comprehension of discharge information, ability to recall discharge information, subjective evaluation of the communication, and patient satisfaction with the admission (scale 1-10 with maximum score 10). Questions addressed admission diagnosis, diagnostic procedures, treatment, follow-up instructions, and when to seek emergency care.
Binary yes/no answers were used to evaluate self-assessed comprehension, eg, "did you understand what was wrong with you/your diagnosis?" The same questions reformulated to obtain descriptive answers were compared to the discharge document in order to evaluate correct recall, eg, "write in your own words what was wrong with you/your diagnosis". Finally, the two answers were compared to evaluate the patients' awareness of comprehension deficits, eg, if a patient answered "yes" to have understood the admission diagnosis but did not recall the correct answer, then the patient was not aware of his comprehension deficit.

In order to validate the questionnaire, authors reviewed answers from the initial ten questionnaires, and since patient answers were as anticipated, the wording of the questions was not changed.

\section{Analysis}

One reviewer (LNH) completed the assessments. However, all uncertainties were discussed and evaluated by the entire group of authors. To minimize bias, objective criteria in scoring the patient responses were established before evaluation.

Patients were given credit for correct recall if they stated the main diagnosis causing their admission in appropriate lay terms, the main diagnostic tests essential for verifying the diagnosis (eg, ultrasound examination for deep venous thrombosis), the newly prescribed medication name or group (eg, antibiotics), and how to take the medication (eg, "once daily" or "twice daily"). Duration of treatment and dosage were not required for a correct answer. However, if these were specified incorrectly, the answer was considered wrong. Particularly in regard to return instructions concerning when to seek emergency care, a relevant answer (eg, shortness of breath after pneumonia) was accepted, even though not documented in the discharge document.

\section{Statistics}

Statistical analyses were performed using SAS software (version 9.2; SAS Institute, Cary, NC, USA). Statistical significance was set as a $P$-value below 0.05 on two-sided tests. Categorical differences between the younger and older group were analyzed with chi-squared or Fisher's exact test if an expected frequency was less than five in any cell of the contingency table. Differences in ordinal categorical variables between groups were analyzed with Cochran-Armitage trend tests, whereas differences in continuous variables were calculated with Wilcoxon rank sum tests and reported as median (interquartile range $[\mathrm{IQR}]$ ) due to skewed distributions in the younger and older group. 
In multiple logistic regression analyses, group differences (older versus younger group) in recall and awareness of comprehension deficits were adjusted for sex and education. Only analyses with a significant likelihood ratio test and no interaction of group with either sex or education were reported. Likewise, the associations between age (continuous variable) and recall as well as awareness of comprehension deficits were investigated when adjusting for sex and education. Models were checked for linearity and potential interaction between age and both sex and education. Only models with a significant likelihood ratio test, no interaction, and linearity were reported.

\section{Results}

A total of 102 patients participated in the study. Forty patients were allocated to the older group and 62 to the younger group (age: 72.0 [IQR: 69-79] years versus 48.5 [IQR: 42-58] years; $P<0.0001)$.

The two groups were comparable in regard to sex (male: $\mathrm{n}=18,45.0 \%$ versus $\mathrm{n}=32,51.6 \% ; P=0.51$ ), highest level of education: primary school, high school, university ( $P=0.06$ ), other diseases (older group: $56.3 \%$ versus younger group: $58.2 \% ; P=0.86$ ), length of admission (1.0 [0-3] days versus $1.0[0-2]$ days; $P=0.97$ ), and patient satisfaction (10.0 [9-10] versus 9.5 [8-10]; $P=0.13)$. More prior admissions, however, were found in the older group $(P=0.027)$.

Admission diagnosis in the older group was mainly infectious illness (22.5\%), musculoskeletal illness (22.5\%), anemia $(20.0 \%)$, neurological illness $(10.0 \%)$, and cardiovascular illness (7.5\%). In the younger group, infectious illness (21.5\%), musculoskeletal illness (18.5\%), neurological illness (12.3\%), back illness (12.3\%), and cardiovascular illness $(9.2 \%)$ were the most common admission diagnoses.

\section{Self-assessed comprehension}

There was no difference in self-assessed comprehension of discharge information between the older and younger group. Most patients answered that they fully understood the information (Table 1) and that the medical staff had used an intelligible language ( $\mathrm{n}_{\text {older }}=36,97.3 \%$ versus $\mathrm{n}_{\text {younger }}=56,94.9 \%$; $P=1.0$ ). Only a few patients thought that their current illness affected their usual understanding $\left(\mathrm{n}_{\text {older }}=3,9.3 \%\right.$ versus $\left.\mathrm{n}_{\text {younger }}=7,12.1 \% ; P=1.0\right)$.

\section{Recall of discharge information}

The older group had more difficulties recalling correct medication instructions (correct recall: $54.3 \%$ versus $78.0 \%$; $P=0.02)$ and diagnostic procedures $(71.8 \%$ versus $91.9 \%$; $P=0.007$ ) compared to the younger group, respectively
Table I Self-assessed comprehension of questions answered yes/no, eg, "did you understand ...?"

\begin{tabular}{llll}
\hline Variable & $\begin{array}{l}\text { Age } \geq \mathbf{6 5} \\
\mathbf{n = 4 0}\end{array}$ & $\begin{array}{l}\text { Age }<\mathbf{6 5} \\
\mathbf{n = 6 2}\end{array}$ & P-value \\
\cline { 2 - 3 } & $\%$ & $\%$ & \\
\hline Admission diagnosis & 97.4 & 98.2 & 1.00 \\
Diagnostic tests & 97.2 & 96.8 & 1.00 \\
Treatment & 100 & 96.7 & 0.53 \\
Preventive measures & 84.4 & 89.8 & 0.51 \\
Medication instructions & 96.6 & 97.7 & 1.00 \\
Follow-up at GP/specialist, why? & 100 & 100 & 1.00 \\
Follow-up at GP/specialist, when? & 100 & 91.9 & 0.27 \\
When to seek emergency care & 96.9 & 92.3 & 0.65 \\
\hline
\end{tabular}

Notes: Individuals who answered "yes" are given as percentages; No differences were found in missing values (\%): age $\geq 65$ : 20 (10.0-26.3) versus age <65: 10.5 (2.4-3।.5); $P=0.49$.

Abbreviation: GP, general practitioner.

(Table 2). The estimated odds for recalling correct medication instructions were 4.2 times higher for the younger group compared to the older group (odds ratio [OR] 4.2, 95\% confidence interval $[\mathrm{CI}] 1.5-11.9, P=0.007)$ when adjusted for sex and education. Furthermore, for each additional year of age the estimated odds for recalling correct medication instructions decreased 6.1\% (OR 0.939, 95\% CI 0.90-0.98, $P=0.001)$ independent of sex and education.

There were no differences between the two groups in recall of the remaining questions, yet several patients in both groups were not able to recall correct information (Table 2).

\section{Awareness of comprehension deficits}

The older group was less aware of their comprehension deficits in four out of seven questions when compared to the younger group; awareness of comprehension deficits with regard to diagnostic tests (older group: $71.1 \%$

Table 2 Recall of discharge information, descriptive answers compared to discharge document

\begin{tabular}{|c|c|c|c|}
\hline Variable & $\begin{array}{l}\text { Age } \\
\geq 65 \text { years } \\
n=40\end{array}$ & $\begin{array}{l}\text { Age } \\
<65 \text { years } \\
n=62\end{array}$ & P-value \\
\hline & $\%$ & $\%$ & \\
\hline Admission diagnosis & 77.5 & 87.1 & 0.20 \\
\hline Diagnostic tests & 71.8 & 91.9 & 0.007 \\
\hline Treatment & 61.5 & 75.4 & 0.14 \\
\hline Preventive measures & 73.7 & 85.7 & 0.24 \\
\hline Medication instructions & 54.3 & 78.0 & 0.016 \\
\hline Follow-up at GP specialist & 75.8 & 77.2 & 0.88 \\
\hline Follow-up at specialist & 79.4 & 93.2 & 0.091 \\
\hline When to seek emergency care & 86.4 & 84.1 & 1.00 \\
\hline
\end{tabular}

Notes: Individuals who answered correctly are given as percentages; No differences were found in missing values (\%): age $\geq 65$ : 13.8 (2.5-31.3) versus age $<65$ : 4.8 (0.8-14.6); $P=0.37$.

Abbreviation: GP, general practitioner. 


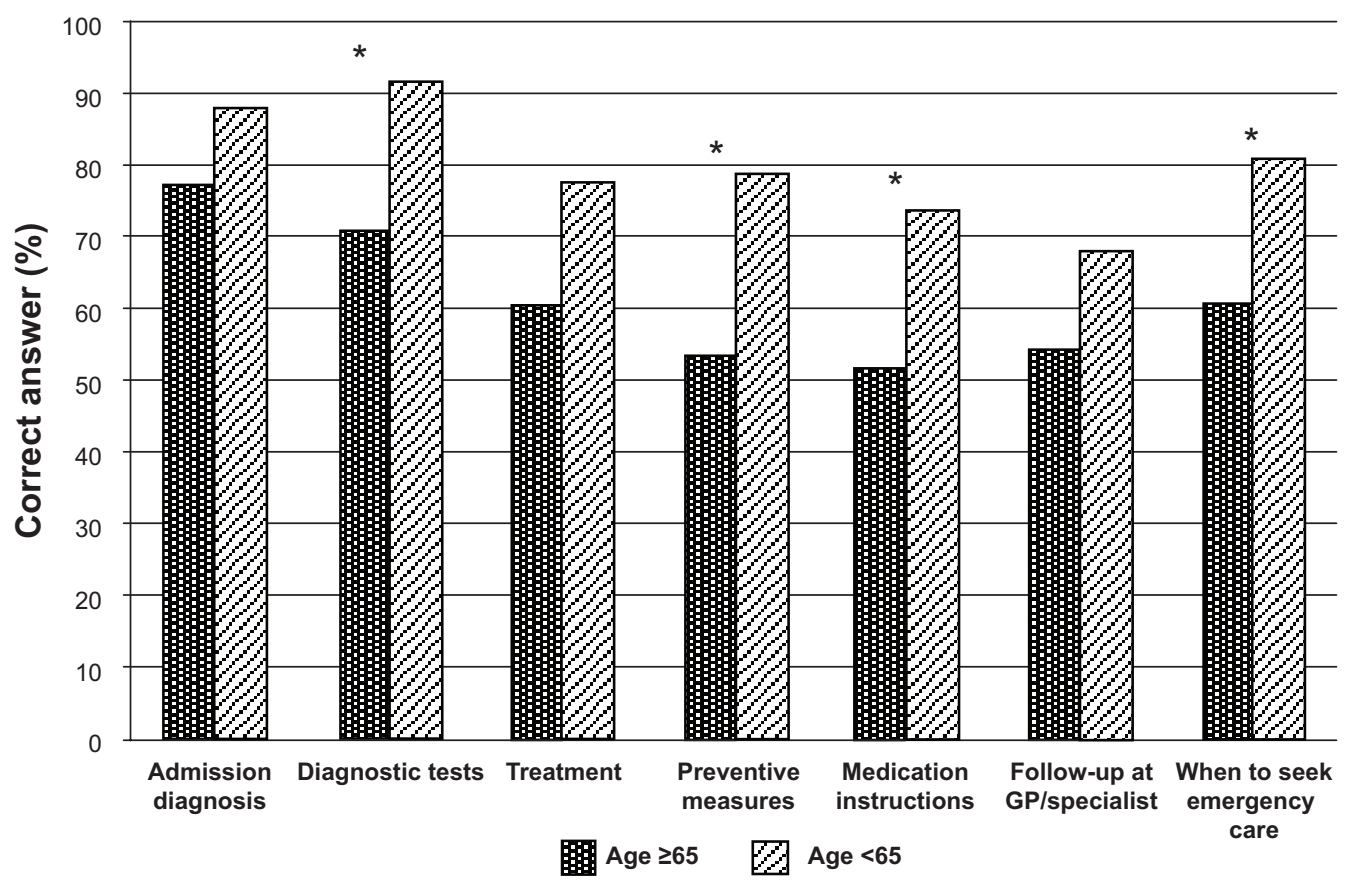

Figure I Awareness of comprehension deficits.

Notes: Comparison of results in Table I and Table 2; Individuals who answered correctly are given as percentages; *Represents level of significance $P<0.05$; No differences were found in missing values (\%): age $\geq 65$ : 7.5 (5.0-17.5) versus age $<65$ : $1.6(0.0-9.7) ; P=0.09$.

Abbreviation: GP, general practitioner.

versus younger group: $91.9 \% ; P=0.006)$, preventive measures $(53.3 \%$ versus $78.6 \% ; P=0.02)$, medication instructions $(51.4 \%$ versus $73.3 \% ; P=0.03)$, and when to seek emergency care $(60.6 \%$ versus $80.8 \% ; P=0.04)$ (Figure 1). The remaining questions showed the same tendency, though not significant: admission diagnosis $(76.9 \%$ versus $88.5 \% ; P=0.12)$, treatment $(60.5 \%$ versus $77.4 \%$; $P=0.07)$, and follow-up at general practitioner/specialist (54.1\% versus $67.7 \% ; P=0.17$ ).

Awareness of comprehension deficits in diagnostic tests was 6.8 times higher among the younger group compared to the older group when adjusted for sex and education (OR 6.8, 95\% CI 1.6-29.2, $P=0.01$ ). Awareness of comprehension deficits in medication instructions and diagnostic tests decreased $6.1 \%$ (OR $0.939,95 \%$ CI $0.904-0.98$, $P=0.001$ ) and $5.8 \%$ (OR 0.94, 95\% CI 0.90-0.99, $P=0.02$ ) per 1-year increase in age, respectively, when adjusted for sex and education. The remaining multiple logistic regression analyses investigating recall and awareness of comprehension deficits were insignificant.

\section{Discussion}

The main finding of the present study was that older patients were less aware of their comprehension deficits when compared to the younger patients. The older patients were unable to identify the misconceived information in four out of seven questions: medication instructions, diagnostic tests, preventive measures, and when to seek emergency care. At the same time, the older patients were less able to recall correct medication instructions and diagnostic tests.

In an internal medicine department and an ED it has been found that patients had difficulties recalling newly prescribed medication. ${ }^{8}{ }^{10}$ Contrary to the present study, patients were not age divided, and these studies were conducted in different settings. In a QDU setting, we found similar recall difficulties, however, most frequently in the older group. Forty-six percent of the older patients did not recall correct medication instructions. This might lead to noncompliance and hereby relapse of illnesses and complications, as potential medication errors have been shown to increase the number of unplanned readmissions. ${ }^{1-4}$ Especially older patients are at risk of medication discrepancies and unplanned readmissions..$^{2-4}$

Despite the comprehension difficulties found in the present study, no QDU readmissions were registered during the 2 months of data collection. However, the limited sample size, along with the relatively short follow-up, limits strong conclusions regarding readmissions. Furthermore, readmission within a short period after discharge indicates more severe illness that could require admission to a more specialized department. 
Knowledge is lacking on older patients' awareness of comprehension deficits, ie, ability to identify the misconceived information. In the present study, we found significant differences between the older and younger patients in four out of eight questions: awareness of comprehension deficits with regard to diagnostic tests, preventive measures, medication instructions, and when to seek emergency care (Figure 1). At the same time, the tendency was that the older group had a lower awareness of comprehension in the remaining questions. A study from an ED found that patients could identify only $20 \%$ of their comprehension difficulties. ${ }^{9}$ These results are difficult to compare to those of the present study since the study design differed considerably. Firstly, only one sample population was described, and no subgroup analysis was conducted. Secondly, the questions were broader, covering major domains such as ED care and post-ED care. Thirdly, analyses were conducted for all questions pooled together and not for each question separately, as in the present study.

The recall difficulties along with the unawareness of comprehension deficits found in the present study emphasize the importance of communicative strategies to confirm patient comprehension before discharge. We did not investigate potential communication failures; however, in a study from an $\mathrm{ED}^{11}$ it was identified that important health information was often missed by the physicians, that patients were rarely asked whether they had questions about the information given, and patient comprehension was never confirmed.

Patients are limited in regard to how much they can process and recall during hospitalization, ${ }^{12,13}$ and many older patients experience a temporary, although recoverable, cognitive dysfunction at discharge.${ }^{14}$ Improvements in this hospitalized related cognitive dysfunction have been found 2 to 4 weeks after discharge, ${ }^{13,14}$ whereas one study found further improvements after 1 year. ${ }^{12}$ In the present study, only $9 \%$ of the older patients thought their illness had affected their comprehension abilities, even though they showed severe difficulties recalling correct discharge information. In this respect, one quarter of the older patients did not recall their potential follow-up at a general practitioner or specialist. At the same time, they were highly unaware of these comprehension deficits. In this respect, we cannot simply rely on the patients to ask the questions needed for clarification or to contact the health care provider in order to secure a sufficient information level.

Optimizing patient comprehension could involve closed loops communication in which the patient repeats the given information. Further, the setting of the discharge interview could be optimized using a single patient room, inviting relatives to join the discharge interview, and implementation of follow-up strategies such as home visits or follow-up calls the day after discharge.

The study has limitations. Firstly, the medical staff were not blinded to the questionnaire, potentially improving the performance of the staff and thereby patient responses. However, patients were approached before leaving the department to avoid recall bias, and it is likely that they may have done better if they had the medication to look at, even though prescription label instructions can be challenging. ${ }^{15}$ Secondly, we did not collect data on mini-mental state examination scores or hearing status at time of admission. Potentially, patients with unknown cognitive and perception impairments could have been included, and this might partly explain the differences in comprehension between the older and younger group. Thirdly, only one reviewer completed the comprehension assessments. Nevertheless, uncertainties were discussed to optimize objective scoring. Fourthly, we did not collect data from other health care levels (eg, primary care) after discharge, and due to the crosssectional design, we are not aware of the clinical consequence of the present results. No readmissions to the QDU were registered during the data-collecting period, but further research is needed to explore this matter.

In a demographic perspective, the older population and number of patients with multiple illnesses are growing. ${ }^{16,17}$ Health care resources are limited, stressing cost-effectiveness and decreasing the length of hospital admissions. ${ }^{18}$ As demonstrated in the present study, older patients represent a vulnerable group in the transition between health care levels. Different follow-up strategies have been introduced to ensure patient compliance after discharge. These involve home visits and telephone follow-up, aimed to encourage patients to assert a more active role during care transitions.

Although there are immediate costs, from an economic prospective, such interventions have shown promising results in lowering the rates of readmission. ${ }^{7,19,20}$

\section{Conclusion}

Older patients were less able to recall correct medication instructions and diagnostic tests when compared to younger patients. Furthermore, the older patients were less aware of their comprehension deficits with respect to medication instructions, diagnostic tests, preventive measures, and when to seek emergency care.

In our perspective, the findings of the present study suggest that communication with the expanding population of older patients requires particular attention. 


\section{Acknowledgments}

We would like to thank all the patients who participated in the present study.

\section{Disclosure}

The authors report no conflicts of interest in this work.

\section{References}

1. Forster AJ, Murff HJ, Peterson JF, Gandhi TK, Bates DW. The incidence and severity of adverse events affecting patients after discharge from the hospital. Ann Intern Med. 2003;138(3):161-167.

2. Marcantonio ER, McKean S, Goldfinger M, Kleefield S, Yurkofsky M, Brennan TA. Factors associated with unplanned hospital readmission among patients 65 years of age and older in a Medicare managed care plan. Am J Med. 1999;107(1):13-17.

3. Corrigan JM, Martin JB. Identification of factors associated with hospital readmission and development of a predictive model. Health Serv Res. 1992;27(1):81-101.

4. Coleman EA, Smith JD, Raha D, Min SJ. Posthospital medication discrepancies: prevalence and contributing factors. Arch Intern Med. 2005;165(16): 1842-1847.

5. McCarthy DM, Waite KR, Curtis LM, Engel KG, Baker DW, Wolf MS. What did the doctor say? Health literacy and recall of medical instructions. Med Care. 2012;50(4):277-282.

6. Baker DW, Gazmararian JA, Williams MV, et al. Functional health literacy and the risk of hospital admission among Medicare managed care enrollees. Am J Public Health. 2002;92(8):1278-1283.

7. Harrison PL, Hara PA, Pope JE, Young MC, Rula EY. The impact of postdischarge telephonic follow-up on hospital readmissions. Popul Health Manag. 2011;14(1):27-32.

8. Maniaci MJ, Heckman MG, Dawson NL. Functional health literacy and understanding of medications at discharge. Mayo Clin Proc. 2008; 83(5):554-558.

9. Engel KG, Heisler M, Smith DM, Robinson CH, Forman JH, Ubel PA. Patient comprehension of emergency department care and instructions: are patients aware of when they do not understand? Ann Emerg Med. 2009;53(4):454-461.e15.
10. Crane JA. Patient comprehension of doctor-patient communication on discharge from the emergency department. J Emerg Med. 1997; 15(1):1-7.

11. Rhodes KV, Vieth $\mathrm{T}, \mathrm{He} \mathrm{T}$, et al. Resuscitating the physician-patient relationship: emergency department communication in an academic medical center. Ann Emerg Med. 2004;44(3):262-267.

12. Inouye SK, Zhang Y, Han L, Leo-Summers L, Jones R, Marcantonio E. Recoverable cognitive dysfunction at hospital admission in older persons during acute illness. J Gen Intern Med. 2006;21(12):1276-1281.

13. Shah MN, Richardson TM, Jones CM, et al. Depression and cognitive impairment in older adult emergency department patients: changes over 2 weeks. J Am Geriatr Soc. 2011;59(2):321-326.

14. Lindquist LA, Go L, Fleisher J, Jain N, Baker D. Improvements in cognition following hospital discharge of community dwelling seniors. J Gen Intern Med. 2011;26(7):765-770.

15. Davis TC, Federman AD, Bass PF, et al. Improving patient understanding of prescription drug label instructions. J Gen Intern Med. 2009;24(1):57-62.

16. Population projections [webpage on the Internet]. Copenhagen: Statistics Denmark. Available from: http://www.dst.dk/en/Statistik/emner/ befolkning-og-befolkningsfremskrivning/befolkningsfremskrivning. aspx. Accessed November 10, 2013

17. Barnett K, Mercer SW, Norbury M, Watt G, Wyke S, Guthrie B. Epidemiology of multimorbidity and implications for health care, research, and medical education: a cross-sectional study. Lancet. 2012; 380(9836):37-43.

18. Oksuzyan A, Jeune B, Juel K, Vaupel JW, Christensen K. Changes in hospitalisation and surgical procedures among the oldest-old: a follow-up study of the entire Danish 1895 and 1905 cohorts from ages 85 to 99 years. Age Ageing. 2013;42(4):476-481.

19. Coleman EA, Parry C, Chalmers S, Min SJ. The care transitions intervention: results of a randomized controlled trial. Arch Intern Med. 2006; 166(17):1822-1828.

20. Phillips CO, Wright SM, Kern DE, Singa RM, Shepperd S, Rubin HR. Comprehensive discharge planning with postdischarge support for older patients with congestive heart failure: a meta-analysis. JAMA. 2004;291(11):1358-1367.
Clinical Interventions in Aging

\section{Publish your work in this journal}

Clinical Interventions in Aging is an international, peer-reviewed journal focusing on evidence-based reports on the value or lack thereof of treatments intended to prevent or delay the onset of maladaptive correlates of aging in human beings. This journal is indexed on PubMed Central, MedLine,

\section{Dovepress}

CAS, Scopus and the Elsevier Bibliographic databases. The manuscript management system is completely online and includes a very quick and fair peer-review system, which is all easy to use. Visit http://www.dovepress. com/testimonials.php to read real quotes from published authors. 